\title{
Evaluation of the capacity limitations and suitability of the European Traffic Management System to support Automatic Train Operation on Main Line Applications
}

\author{
P. Thomas ${ }^{1}$, D. Fisher ${ }^{1} \&$ F. Sheikh ${ }^{2}$ \\ ${ }^{1}$ Parsons Group International Ltd, UK \\ ${ }^{2}$ Department for Transport, UK
}

\begin{abstract}
The European Rail Traffic Management System (ERTMS) is the concept by which Europe is moving towards the standardisation of its rail signal control systems. Standardisation focuses on interfaces necessary for interoperability between trainborne and trackside equipment. ERTMS represents a step change for many railways', the vast majority of which are signalled with colour light signals and basic warning systems. ERTMS is specified in a number of different levels, and dependant on the implementation can introduce the benefits of cabsignalling, automatic train protection and future moving block train separation.

This paper examines the capabilities and limitations of an ETCS/ERTMS level 2 implementation as specified by the current Technical Specifications for Interoperability for use in high density network locations and the suitability of ETCS/ERTMS to support the integration of an Automatic Train Operation (ATO) overlay. A generic sample section has been developed to analyse the headway constraints within the current ERTMS solution. The conclusions from the study suggest that there are no fundamental constraints preventing a level 2 implementation supporting an operational headway of 24 Trains Per Hour with recovery margin. The use of ATO for a high density application will offer an improved headway performance but will require a level of development and enhancement to the ERTMS functionality and architecture to correctly implement some ATO functionality. A further potential constraint is the capacity of the GSM-R radio communication network. This limitation can be removed by the envisaged migration to packet mode (GPRS) data transmission.
\end{abstract}

Keywords: ERTMS, systems performance, train control systems, GSM-R, ATO. 


\section{ERTMS implementation in the UK}

The European Rail Traffic Management System (ERTMS), including the European Train Control System (ETCS) have been developed under the umbrella of the EU Interoperability Directives to facilitate rail interoperability throughout Europe, and to realise the benefits of standardised solutions, and volume procurement in terms of pricing. In recognition of the value, and importance of moving towards standardised systems, the UK has committed itself to the deployment of ETCS, and it will be fitted as the replacement signalling system on asset renewal.

ETCS seeks to address a range of operating conditions, and needs throughout Europe, but was originally developed with emphasis on high speed operation. It offers three functional levels:

Level 1: Overlay to existing signalling system; movement authority through Eurobalise; train integrity and position by track circuit.

Level 2: Cab based signalling; movement authority through GSM-R; train position via Eurobalise; train integrity/occupancy through track-circuits/axle counters.

Level 3: Cab based signalling; movement authority through GSM-R; train position via Eurobalise; train integrity on-board; potential for moving block.

The system planned for deployment in the UK is Level 2 without line side signal back-up: a full communications based cab-based signalling system. To date, ERTMS/ETCS Level 2 has been implemented on high speed lines within the European Union, and is starting to be implemented on mixed traffic main line railways. In the UK the deployment of ETCS will become a routine activity, relying upon a standardised approach to procurement, system configuration, and installation.

\section{Modelling ETCS capability}

Within the UK ETCS must be capable of providing signalling control for various traffic and operational scenarios, including high density suburban systems such as the new London Crossrail system. Today, such high density suburban systems are also considering the use of Automatic Train Operation (ATO) systems to ensure that their timetable demands can be reliably met.

To better understand the limits of ETCS performance, the Department for Transport commissioned a study into an analysis of its capability, and also to determine whether there were any constraints within the system as specified that would prevent it from being integrated with an ATO system.

\section{Methodology and basis}

A bespoke model (CPetcs) was developed to assess the headway constraints by modelling the train throughput capacity through a generic sample section, equipped with an ETCS level 2 application without visual signals. The model enables the dynamic behaviour of an ETCS application to be evaluated for 
Table 1: $\quad$ Basis.

\begin{tabular}{|l|}
\hline Data \\
\hline $\begin{array}{l}\text { EEIG ERTMS USERS GROUP: 97E881 Version 6L Description of the brake } \\
\text { curve calculation [R1] }\end{array}$ \\
\hline ERTM/CAM/D0128 Issue 2.1 System Description (National Values) [R2] \\
\hline IEP-TECH-REQ-35 Issue 01 Train Technical Specification [R3] \\
\hline ATO Data [R4] \\
\hline Electrostar train data [R4] \\
\hline ETCS SRS Class 1 version 2.3.2 [R5] \\
\hline
\end{tabular}

different scenarios and configurations of the ETCS application including with an ATO overlay. The data used in the model is summarised in Table 1.

\section{Railway model}

The CPetcs model development is based on the following principles: The number of trains per hour that can be scheduled to run over a particular railway section depends on the minimum headway, between two consecutive trains that can be achieved at the worst bottleneck within the section; The model therefore simulates the running of a number of trains separated by, user defined target headways over a, user defined sample railway section; The speed profiles of these trains is dependent on train performance characteristics, Permanent Speed Restrictions (PSR), gradients, station stopping points and dwell times, all of which are user inputs to the model; Constraints imposed by ERTMS / ETCS signalling are then imposed by the model in accordance with interoperability specifications (TSI) [R1,R2,R5]; The extent of the signalling constrains depends on the following factors (all of which are included in the model):

- Operations of trainborne ETCS equipment with, user defined, performance characteristics based on TSI values [R2,R5].

- Operations of trackside ETCS equipment with, user defined, performance characteristics. Values of these characteristics are based on Parsons experience of typical supplier capabilities.

- Delays in message interchange between the two due to the GSM-R network. These are based on TSI values [R5] of message lengths and GSM-R performance requirements.

- Braking curve calculations as specified by EEIG [R1].

The margins between target and achievable headways are computed by the model in order to indicate the feasibility of achieving the target capacity, and the level of margin available for schedule recovery with and without ATO.

\subsection{Headway scenarios analysed}

The following scenarios have been analysed:

a. A plain line section with gradient change from uphill to downhill;

b. The same plain line section with the inclusion of an Radio Block Centre (RBC) boundary; 
c. A section including a station stop;

d. A section including a converging junction;

e. A section including a diverging junction;

f. A composite section including all the above features together with PSR changes.

\subsubsection{Scenario parameter values}

All scenarios use the following parameter values: Electrostar Class 375 train; Manual driving - $90 \%$ of full braking, $98 \%$ of ceiling speed, $99 \%$ of acceleration; Axle Counters are used for train detection.

For scenarios (a) and (b) the following parameter values have been used: Scheduled headway 75 seconds; $10 \mathrm{~km}$ plain line track; Line speed $120 \mathrm{kph}$; Initial gradient $1 \%$ uphill changing to $1 \%$ downhill after $5 \mathrm{~km} ; 8$ car train

Other key parameters were varied according to Table 2 Headway Key Parameters. GSM-R "Circuit Switched Mode (CSM) high error" represents a 10 fold increase in random errors and call drop outs and GSM-R cell size reduction from $4 \mathrm{~km}$ to $2 \mathrm{~km}$ (i.e. double frequency of hand-over gaps).

Table 2: $\quad$ Headway key parameters.

\begin{tabular}{|l|c|c|c|}
\hline Variable & Better & Nominal & Worse \\
\hline Block size $(\mathrm{m})$ & 400 & 500 & 600 \\
\hline Balise space $(\mathrm{m})$ & 100 & 400 & 1200 \\
\hline Train length (Class 375) & $4 \mathrm{car}$ & $8 \mathrm{car}$ & $12 \mathrm{car}$ \\
\hline ATP / ATO & ATO & ATP & ATP \\
\hline MA event/cycle & event & event & cycle \\
\hline GSM-R & GPRS mean & CSM 99\% & $\begin{array}{l}\text { CSM 99\% } \\
\text { high error }\end{array}$ \\
\hline
\end{tabular}

\subsection{ETCS level 2 capacity analysis results}

The results give an indication of the impact of different railway features on the capacity of a line section. They also indicate which signalling related parameters have greatest impact on capacity. In broad terms they indicate a capacity limit of about 24 tph for ETCS Level 2 with manual driving. However a particular railway layout can contain features that interact to further constrain its capacity. This implies the need to analyse real line sections before drawing final conclusions on their capacity.

\subsubsection{Results for plain line scenarios (a) \& (b)}

The results, in terms of maximum trains per hour, are shown on fig. 1. Each axis in the radar diagram shows capacity sensitivity to a specific parameter value.

The following observations can be made on these results (taking the parameters in a clockwise sequence):

- $\quad$ ETCS Block size has a very significant impact. The values illustrated (400 m, $500 \mathrm{~m}$ and $600 \mathrm{~m}$ ) were chosen to be relatively close to avoid 
distorting the overall chart. Larger block sizes show a marked reduction in railway capacity but there is a law of diminishing returns as block sizes decrease.

- Balise spacing has a relatively small impact.

- The length of the train is a significant factor. The significance increases at low train speeds.

- ATO offers a very significant improvement.

- The TSI specifications allow two principal options for delivering Movement Authority updates to trains. Results show that the RBC should send updates as soon as available (event option) rather than waiting for cyclic repeat requests from the train (cycle).

- The nominal values are all based on GSM-R operating in Circuit Switched Mode and performing in accordance within specified limit. Higher values of error rates, call dropout rates and cell handover gaps can have a significant adverse effect on capacity. GPRS offers a modest improvement.

- There is a relatively small deterioration in headways as trains cross $\mathrm{RBC}$ boundaries due to the time for inter-RBC communication.

\subsubsection{Results for station stops (c)}

The impact of station stops depends largely on the dwell time as illustrated in fig. 2.

\subsubsection{Results for converging junctions (d)}

The impact of a converging junction as a function of the turn-in speed limit compared to the $120 \mathrm{kph}$ points-normal line speed is shown in fig. 3 . The impact is modest. The irregularity is caused by interaction with the block spacing.

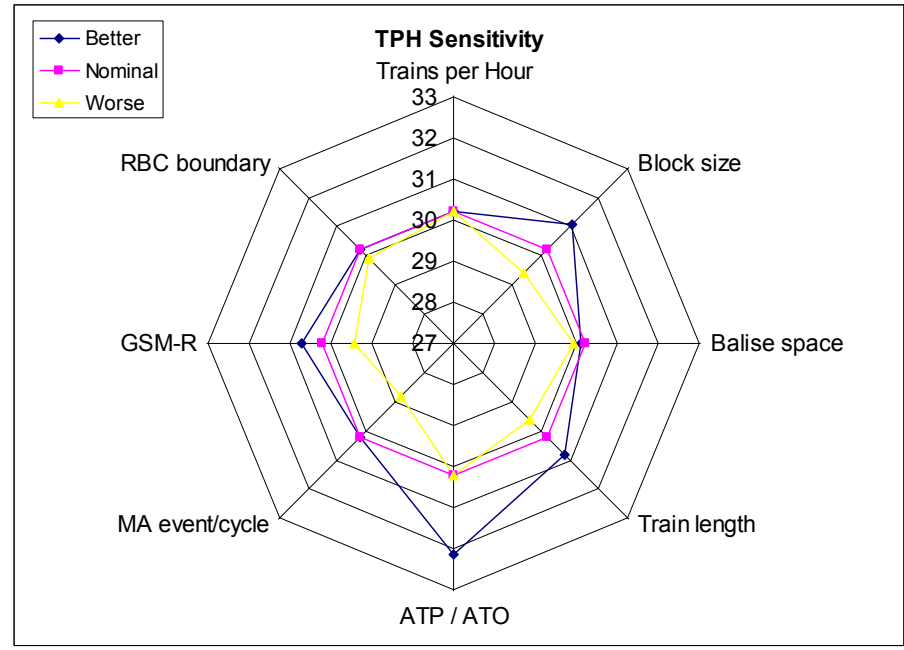

Figure 1: Results of plain line analysis. 




Figure 2: $\quad$ Capacity reduction from station stop.



Figure 3: $\quad$ Capacity reduction from turn-in speed.

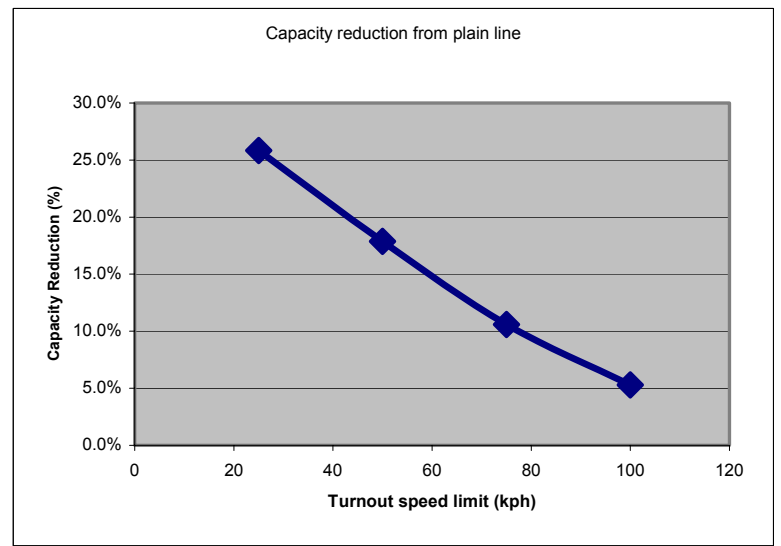

Figure 4: $\quad$ Capacity reduction from turnout speed. 


\subsubsection{Results for diverging junctions (e)}

The diverging junction scenario (e), however, shows a much more significant capacity impact as illustrated in fig 4 compared with a points-normal speed limit of $120 \mathrm{kph}$.

\subsubsection{Results for the sample section (f)}

A headway plot for a hypothetical railway sample section is shown below.

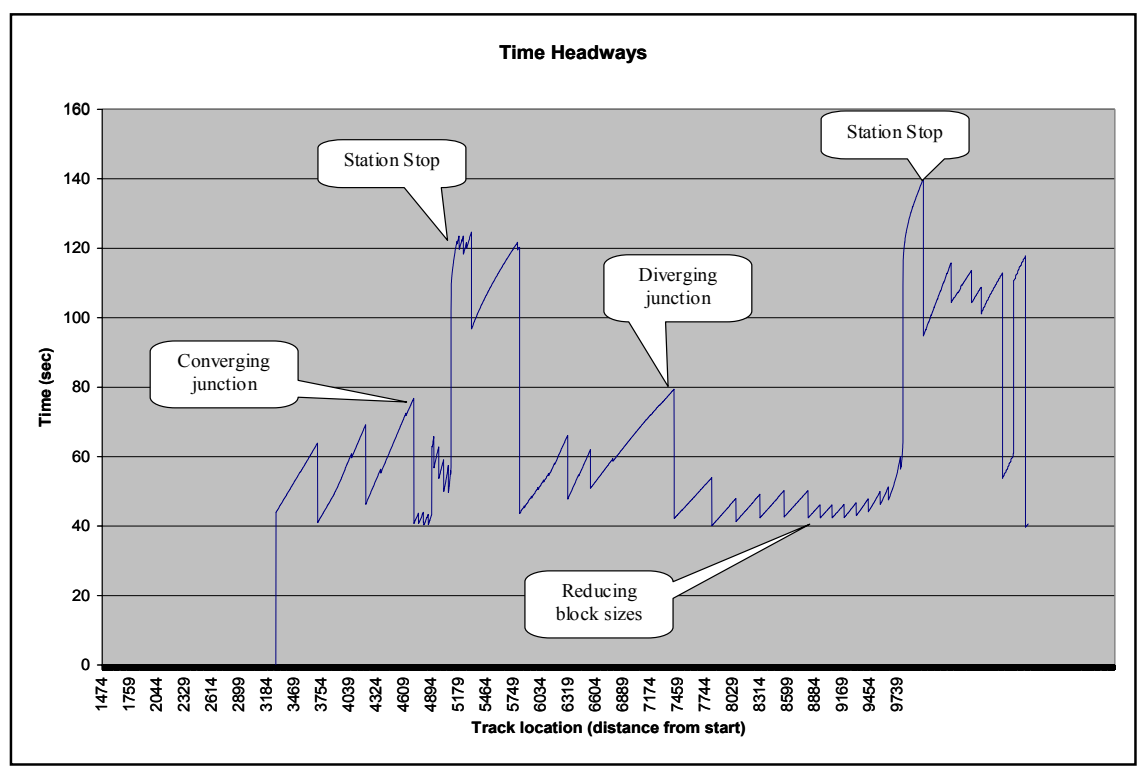

Figure 5: Sample section results.

The shape of the headway plot can be explained as follows:

- The trace starts at a point about $3300 \mathrm{~m}$ from the start of the specimen section. This is the location of the leading train when the following train starts its journey.

- Headways increase as the following train accelerates because the braking time increases.

- The sharp drops in headway coincide with the lead train passing an overlap and hence freeing up the preceding block.

- There is an increase in headway at the converging junction (marked).

- The headway drop after the converging junction is due to the following train braking to a PSR reduction.

- The converging junction precedes a station stop and short block spacing has been provided in these areas. This explains the small headway fluctuations followed by a sharp increase when the lead train stops at the station.

- The headway then drops as the lead train leaves the station.

- It then rises again as the lead train slows down for a diverging junction. 
- Following the diverging junction we see the effect of varying block sizes.

- The final rise is for a second station stop. This rise is more pronounced as the following train is at full line speed in this case

\section{ATO integration analysis}

To assess whether ERTMS could be integrated successfully with an ATO overlay the interactions between the on-board elements of the ETCS system and the ATO are considered by analysing the data flows, represented by arrows, identified in the ATO functional model presented in fig. 6 below. For the purposes of the analysis the model defined in IEEE P1474.3 has been adapted for an ERTMS application. The model summarises the interfaces between ATO sub-functions (solid boxes) to non ATO sub-functions (dashed boxes). Within each box the system performing the function is indicated by: "[A]" performed by ATS equipment, "[W]" by ETCS wayside equipment, "[T]" by ETCS/ATO train-borne equipment, "[E]" indicates by external equipment to ETCS/ATO, "[W/T]" by either wayside equipment or train-borne equipment, depending on the specific ETCS system design, "[T/E]" by either train-borne or external equipment, depending on the specific ETCS system design.

The station stopping functionality of ATO is modelled by analysing the affects of the odometry accuracy on the performance of ATO to regulate its train operations profile.

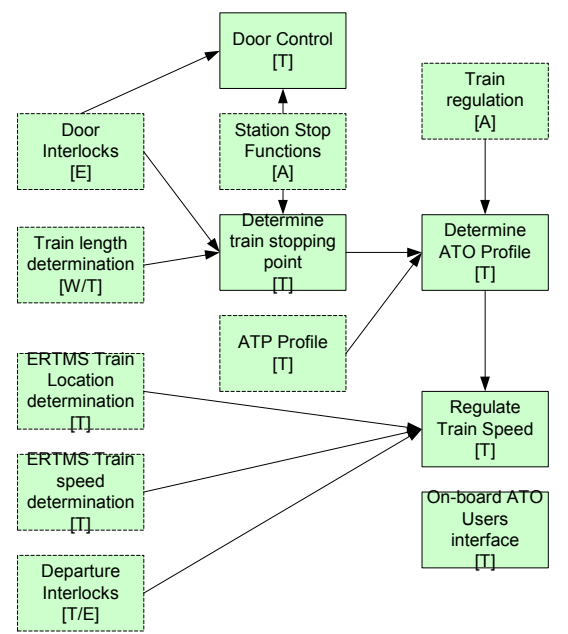

Figure 6: $\quad$ ATO functional model.

\subsection{Results}

\subsubsection{ATO fixed data}

The ATO requires fixed data to perform its functions such as calculate ATO profile, Door Control and station stopping. In an ERTMS application the 
movement authority sent to the train includes some of the information required by the ATO to perform these functions. The data may therefore be transmitted by the on-board EVC to the ATO or held within the ATO system as part of the fixed data. Whether the fixed data elements are sent to the ATO at the same time that the EVC receives an MA update or the data is held within the ATO makes no significant difference to the performance of the ATO overlay and does not present an integration issue.

\subsubsection{ERTMS train location determination}

This function provides the train location information to the ATO to enable the ATO to control the train speed and stop the train accurately at the platform. The accuracy of the platform stop will be governed largely by the accuracy of the odometry system. The odometry system uses the balises to provide a positional reference. The ATO system uses this information to update and correct its location information used to regulate the train speed, which may result in the ATO modifying its propulsion or brake commands. During a platform stop the effects of two cases of odometry error are considered: In the first case there is an odometry error, which causes the ATO to believe that the train has not travelled as far as the actual train position. The ATO upon receipt of a location update after reading the balise will release the service brake in an attempt to follow the correct stopping curve. Once the ATO is following the correct stopping curve it will re-apply the service brake. The effects of the brake-build-up time will cause an overshoot of the stopping point. If no correction is made the train will stop short or undershoot the stopping location. In the second case an odometry error causes the ATO to believe that it has travelled further than it actually has. In this case as the ATO is already demanding full service brake the train will continue to overshoot the platform stopping position.

\section{Conclusions}

The conclusions from the study suggest that there are no fundamental constraints preventing an ETCS/ERTMS level 2 implementation supporting a technical headway of 120 seconds or better, enabling an operational headway of 24 Trains Per Hour with 30 seconds recovery margin. The implementation of ATO for a high density application offers a significant improvement to headway performance and will enable a higher train capacity throughput to be sustained. The implementation of an ATO overlay to ETCS /ERTMS will require a level of development and enhancement to the ERTMS functionality and architecture to correctly implement some ATO functionality that maybe desirable in certain applications. These developments are largely to implement ATO station stopping functionality.

It can be further concluded that it should be possible to overlay an ATO system to an ETCS application. The accuracy of the odometry system would need to be sufficient to support the correct function of the ATO. The solution and performance is likely to be supplier specific.

A further potential constraint is the capacity of the GSM-R radio communication network for communication between ETCS Level 2 trainborne 
and trackside signalling equipment. The present versions of TSIs require an individual radio channel for each ETCS controlled train. The available GSM-R bandwidth could become a limitation. This limitation can be removed by the, envisaged, migration of the ETCS standards to include packet mode (GPRS) data transmission.

Although not embodied within the TSI's, the TSI's themselves do not prevent implementation of an ATO overlay as analysed in this study, for example the TSI's do not prevent implementation of a more accurate odometry system. The authors are also aware that there are number of change requests proposed that could if implemented enable some ATO functionality such as door control and standard automatic driving interface. The TSI's will however, need to be modified to implement GPRS. This would also require development of the necessary ETCS functionality.

\section{References}

[1] Network Rail National ERTMS Programme Team, EEIG ERTMS USERS GROUP: 97E881 Version 6L Description of the brake curve calculation

[2] Network Rail National ERTMS Programme Team, ERTM/CAM/D0128 Issue 2.1 System Description (National Values)

[3] DfT IEP-TECH-REQ-35 Issue 01 Train Technical Specification

[4] Parsons, ATO data and Electrostar data

[5] ERA website, ETCS SRS Class 1 version 2.3.2 